\title{
Therapeutic Effects of Extinction Learning as a Model of Exposure Therapy in Rats
}

\author{
Elizabeth A Fucich', Denisse Paredes' and David A Morilak*,I \\ 'Department of Pharmacology and Center for Biomedical Neuroscience, University of Texas Health Science Center at San Antonio, San Antonio, \\ TX, USA
}

\begin{abstract}
Current treatments for stress-related psychiatric disorders, such as depression and posttraumatic stress disorder (PTSD), are inadequate. Cognitive behavioral psychotherapies, including exposure therapy, are an alternative to pharmacotherapy, but the neurobiological mechanisms are unknown. Preclinical models demonstrating therapeutic effects of behavioral interventions are required to investigate such mechanisms. Exposure therapy bears similarity to extinction learning. Thus, we investigated the therapeutic effects of extinction learning as a behavioral intervention to model exposure therapy in rats, testing its effectiveness in reversing chronic stress-induced deficits in cognitive flexibility and coping behavior that resemble dimensions of depression and PTSD. Rats were fear-conditioned by pairing a tone with footshock, and then exposed to chronic unpredictable stress (CUS) that induces deficits in cognitive set-shifting and active coping behavior. They then received an extinction learning session as a therapeutic intervention by repeated exposure to the tone with no shock. Effects on cognitive flexibility and coping behavior were assessed $24 \mathrm{~h}$ later on the attentional set-shifting test or shock-probe defensive burying test, respectively. Extinction reversed the CUS-induced deficits in cognitive flexibility and coping behavior, and increased phosphorylation of ribosomal protein S6 in the medial prefrontal cortex (mPFC) of stress-compromised rats, suggesting a role for activity-dependent protein synthesis in the therapeutic effect. Inhibiting protein synthesis by microinjecting anisomycin into mPFC blocked the therapeutic effect of extinction on cognitive flexibility. These results demonstrate the utility of extinction as a model by which to study mechanisms underlying exposure therapy, and suggest these mechanisms involve protein synthesis in the mPFC, the further study of which may identify novel therapeutic targets.

Neuropsychopharmacology (2016) 4I, 3092-3102; doi:I0.1038/npp.2016.127; published online I0 August 2016
\end{abstract}

\section{INTRODUCTION}

Stress-related psychiatric illnesses, including depression, posttraumatic stress disorder (PTSD), and anxiety disorders, are highly comorbid and share several symptom dimensions, including cognitive dysfunction and maladaptive coping strategies, and common risk factors, including chronic stress. Thus, these disorders are likely to share common neurobiological substrates (Biehn et al, 2013; Elhai et al, 2011). Existing pharmacotherapies are of limited effectiveness, and treatment resistance and relapse remain problematic (Janicak et al, 1997; Nutt, 2010). Cognitive behavioral psychotherapies, including exposure therapy, are effective for some pharmacotherapy-resistant patients, and the two approaches can be combined for greater effect (Beck, 2005; Hollon et al, 2002; Nemeroff et al, 2003; Wiles et al, 2013). However, the efficacy of psychotherapy is also limited (de Kleine et al, 2013), and outcomes remain unacceptably poor. Thus, a better understanding of neural mechanisms underlying

*Correspondence: Dr DA Morilak, Department of Pharmacology, University of Texas Health Science Center, Mail Code 7764, 7703 Floyd Curl Drive, San Antonio, TX 78229-3900, USA, Tel: + I 210567 4I74, Fax: + I 210567 4300, E-mail: Morilak@uthscsa.edu

Received 26 January 20 I6; revised 17 June 20 I 6; accepted I I July 20 I6; accepted article preview online 15 July 2016 effective psychotherapy could guide the development of more effective treatments.

Cognitive dysfunction, including perseverative thinking and cognitive inflexibility, is a major component of these disorders and may not only be a symptom, but also underlie onset and maintenance of illness (Beck, 1976; Coles and Heimberg, 2002; Mathews and Mackintosh, 1998). Cognitive flexibility is an executive process that enables one to modify preestablished thoughts and strategies based on feedback from a changing environment (Beck, 1976). Hypoactivity in the medial prefrontal cortex (mPFC), which mediates cognitive flexibility and regulates coping strategy selection, is a prominent feature of these disorders (Rogers et al, 2004; Sheline, 2003). Cognitive behavioral therapies are intended to improve cognitive flexibility, to ameliorate perseverative, negatively biased thought (Beck, 2005; Brewin, 1996), and improve adaptive responding. Neuroimaging studies have demonstrated $\mathrm{mPFC}$ activation in psychotherapy patients (Ritchey et al, 2011; Straub et al, 2015; Yoshimura et al, 2014). Thus, mPFC activation may be important for therapeutic efficacy.

Chronic unpredictable stress (CUS) impairs cognitive flexibility in rats, producing a deficit in extradimensional set-shifting on the attentional set-shifting test (AST) (Bondi et al 2008, 2010; Jett and Morilak, 2013), mediated by the 
Table I CUS Schedule

\section{CUS schedule for experiment I}

$\begin{array}{llll}\text { Day - I } & \begin{array}{l}\text { Habituation to fear } \\ \text { conditioning contexts }\end{array} & \text { Day 9 } & \text { Warm swim } \\ \text { Day 0 } & \text { Fear conditioning (all groups) } & \text { Day 10 } & \text { Social defeat } \\ \text { Day I } & \text { Restraint } & \text { Day 1 I } & \begin{array}{l}\text { Cold stress (begin food } \\ \text { restriction for AST) }\end{array} \\ \text { Day 2 } & \text { Shaking/crowding } & \text { Day 12 } & \text { Tail pinch } \\ \text { Day } 3 & \text { Social defeat } & \text { Day 13 } & \text { Cold swim } \\ \text { Day } 4 & \text { Warm swim } & \text { Day 14 } & \text { Cold stress } \\ \text { Day } 5 & \text { Wet bedding } & \text { Day 15 } & \text { AST habituation } \\ \text { Day } 6 & \text { Social defeat } & \text { Day 16 } & \text { AST training } \\ \text { Day 7 } & \text { Shaking/crowding } & \text { Day 17 } & \text { Extinction training } \\ \text { Day } 8 & \text { Cold stress } & \text { Day 18 } & \text { AST testing }\end{array}$

CUS schedule for experiments 2-5

\begin{tabular}{|c|c|c|c|}
\hline Day - I & $\begin{array}{l}\text { Habituation to fear } \\
\text { conditioning contexts }\end{array}$ & Day 9 & Warm swim ${ }^{a}$ \\
\hline Day 0 & $\begin{array}{l}\text { Fear conditioning } \\
\text { (extinction groups only) }\end{array}$ & Day 10 & Social defeat \\
\hline Day I & Restraint & Day II & $\begin{array}{l}\text { Footshock (begin food } \\
\text { restriction for AST) }\end{array}$ \\
\hline Day 2 & Shaking/crowding & Day 12 & Tail pinch \\
\hline Day 3 & Social defeat & Day 13 & Cold swim ${ }^{a}$ \\
\hline Day 4 & Warm swim ${ }^{\mathrm{a}}$ & Day 14 & Cold stress \\
\hline Day 5 & Wet bedding & Day 15 & AST habituation \\
\hline Day 6 & Social defeat & Day 16 & AST training \\
\hline Day 7 & Shaking/crowding & Day 17 & $\begin{array}{l}\text { Extinction training or } \\
\text { tone control treatment }\end{array}$ \\
\hline Day 8 & Footshock & Day 18 & SPDB or AST testing \\
\hline
\end{tabular}

${ }^{\mathrm{a}} \mathrm{S}$ wim stress was replaced by other stressors in experiment 5 to avoid infection due to surgery.

mPFC (Birrell and Brown, 2000). In the shock-probe defensive burying (SPDB) test, CUS induces a shift from active to passive coping (Jett et al, 2015) that resembles maladaptive avoidance behaviors seen in many stress-related psychiatric illnesses (Bondi et al, 2007; Koolhaas et al, 1999). Behavioral response on the shock-probe test is mediated in the lateral septum (LS) (Bondi et al, 2007; Koolhaas et al, 1999; Treit et al, 1993), and modulated by the MPFC (Shah et al, 2004). Both the CUS-induced cognitive impairment and shift toward passive coping are reversed by pharmacological interventions with antidepressant drugs (Bondi et al, 2008, 2010; Jett et al, 2015). In this study, we tested whether a cognitive behavioral intervention, extinction learning, has similar therapeutic effects on CUS-induced deficits in cognitive flexibility and coping.

Fear extinction bears many similarities to exposure therapy, a cognitive behavioral therapy for PTSD that involves repeated exposure to conditioned fear-provoking stimuli in a nonthreatening environment until fearful responding decreases (Foa and Meadows, 1997). This can be modeled in rats by first conditioning them to fear an innocuous cue (tone) by pairing it with an aversive stimulus (mild shock). Extinction then involves repeated exposure in a different context to the conditioned cue alone, with no shock, producing new learning that the cue no longer signals danger in that context, resulting in suppression of fear (Quirk et al, 2006). Like cognitive behavioral therapies in humans, extinction learning activates the mPFC in rats and modulates the activity of downstream targets that mediate fear behavior (Sotres-Bayon et al, 2004). Similarities in procedure and neurocircuitry between extinction and exposure therapy have been noted (Hoffmann, 2008; McNally, 2007), but the effectiveness of extinction as a preclinical model of therapeutic intervention has not been demonstrated. Such a model would allow the study of neural mechanisms underlying the pathology of stress-related illnesses and novel mechanisms of treatment.

In this study, we tested the utility of fear extinction as a behavioral intervention modeling exposure therapy following CUS. After demonstrating a beneficial effect in reversing CUS-induced behavioral deficits, we began to explore mechanisms by which extinction may improve stresscompromised function of the $\mathrm{mPFC}$. Long-term retention of extinction memory requires new protein synthesis in the mPFC (Santini et al, 2004). We measured phosphorylation of ribosomal protein S6 in stressed and unstressed rats after extinction as an indicator of changes in activity-dependent protein synthesis, an important process in synaptic plasticity (Buffington et al, 2014; Richter and Klann, 2009). We then tested whether de novo protein synthesis in the MPFC was necessary for the therapeutic effect of extinction on cognitive flexibility.

\section{MATERIALS AND METHODS}

\section{Animals}

A total of 210 adult male Sprague-Dawley rats (Harlan, Houston, TX), 220-240 g, were individually housed on a 12/12-h light/dark cycle (lights on at $0700 \mathrm{~h}$ ), with food and water ad libitum. For social defeat, 12 male Long-Evans rats, 400-450 g (Charles River, Wilmington, MA), were pair-housed with ovariectomized females in large cages $(63 \times 63 \times 40 \mathrm{~cm})$. Experiments were conducted during the light phase. All procedures were in accordance with $\mathrm{NIH}$ guidelines and approved by the UTHSCSA institutional animal care and use committee.

\section{Chronic Unpredictable Stress}

As previously described (Bondi et al, 2008), a different acute stressor was applied each day (Table 1$)$. In experiment 1 , footshock was replaced with cold stress $\left(4^{\circ} \mathrm{C}, 6 \mathrm{~h}\right)$ to avoid potential interference with the unconditioned footshock used for fear conditioning. In experiment 2, including footshock in the CUS did not affect extinction. As that also produces a more robust stress effect, the second CUS protocol in Table 1 was used in experiments $2-5$. Swim stress was replaced with other stressors in experiment 5 to avoid infection after surgery. Unstressed controls were handled daily.

\section{Extinction Treatment}

Day -1: context habituation. At 2 days before beginning CUS, rats were habituated to two contexts in sound-attenuating 
cabinets for $15 \mathrm{~min}$ each. Context $\mathrm{A}$ was the conditioning chamber $(30.5 \times 25.4 \times 30.5 \mathrm{~cm}$; model H10-11R-TC, Coulbourn Instruments, Whitehall, PA) with metal walls and a metal grid floor attached to a shock generator (model H13-15). Context B was a different chamber, with smooth green vinyl floor and circular walls.

Day 0: fear conditioning. To avoid CUS altering fear conditioning, rats were fear-conditioned in context A the day before beginning CUS, with 4 pairings of a tone $(10 \mathrm{kHz}$, $75 \mathrm{~dB}, 20 \mathrm{~s})$ coterminous with footshock (0.8 mA, $0.5 \mathrm{~s})$. Average intertrial interval (ITI) was $120 \mathrm{~s}$. Conditioned fear was expressed as percent freezing during each tone, measured videographically, and defined as movement falling below the index threshold for at least $1 \mathrm{~s}$ (FreezeView software, ActiMetrics \#ACT-100, Coulbourn). Subjects were then assigned to groups based on freezing during tone 4, such that initial freezing was roughly comparable.

Day 17: extinction. At 3 days after the end of CUS, rats underwent a single extinction session in context $\mathrm{B}$, consisting of 16 presentations of tone alone with no shock (average ITI $120 \mathrm{~s}$ ). Freezing was typically maximal on the second extinction trial $(\sim 70 \%)$, decreasing over $7-10$ trials to a final level of $\sim 25 \%$ (Green et al, 2011). In experiment 1, nonextinction controls were fear-conditioned but did not receive extinction training on day 17 to ensure that fear conditioning itself did not affect AST performance. In experiments $2-5$, all rats underwent the extinction procedure on day 17. Controls did not receive prior fear conditioning, thus controlling for potential effects of environmental enrichment by exposure to tones in context $\mathrm{B}$, independent of extinction learning. We refer to these as 'tone controls' to distinguish them from the controls in experiment 1.

\section{Experiment 1: Effect of Extinction Training as a Therapeutic Intervention on the CUS-Induced Deficit in Cognitive Flexibility on the AST}

A total of 64 rats were used in 4 groups, defined by Stress (CUS or control) and Extinction (extinction or nonextinction control). All rats were fear-conditioned on day 0. CUS or nonstress control treatment began the day after conditioning. For rats undergoing extinction, it was conducted on day 17, 3 days after the end of CUS. All rats were tested on the AST on day 18 (Figure 1a).

Attentional set-shifting test. Cognitive flexibility was measured on the extradimensional (ED) set-shifting task of the AST, as previously described (Bondi et al, 2008; Jett and Morilak, 2013; Lapiz and Morilak, 2006). Beginning on day 11 of CUS, rats were food restricted to $14 \mathrm{~g} /$ day. The test requires 3 days as follows.

Day 15: habituation. At 1 day after the end of CUS, rats were taught to dig for food reward (1/4 Honey Nut Cheerio, General Mills, Minneapolis, MN) in terracotta pots filled with sawdust, first in their home cage and then in the testing arena.
Day 16: training. Rats learned to make simple discriminations, first by associating the reward with one of two odors (lemon vs rosewood-scented pots, both filled with sawdust) and then with one of two digging media (unscented pots filled with felt $v s$ paper).

Day 18: testing (24h after extinction). Half the rats were tested using odor as the informative cue to form a 'cognitive set' in the first five test stages. The other half were tested using medium as the informative cue in the initial stages that comprised: simple discrimination, complex discrimination, reversal learning, new acquisition, and second reversal, all with the same relevant stimulus dimension (odor or medium). In the ED set-shift, the previously irrelevant dimension became relevant and the previously relevant dimension became the distractor, requiring the rat to shift attention from one stimulus dimension to the other. Testing on each task continued to a criterion of six consecutive correct trials before proceeding to the next. The measure of cognitive flexibility was the number of trials to criterion (TTC) on the ED task. Behavior was scored by experimenters blind to treatment groups.

\section{Experiment 2: Replication of the Therapeutic Effect of Extinction Training on Cognitive Flexibility Using CUS with Footshock and Using Controls Exposed to the Extinction Chamber and Tones}

The purpose of this experiment was to replicate the extinction-induced improvement in stress-compromised cognitive flexibility in experiment 1, using a more robust CUS procedure that includes footshock, and employing a control for environmental enrichment from exposure to context B plus tones, independent of extinction learning. A total of 11 rats were used in 3 groups, defined by Stress (CUS or control) and Extinction (extinction or tone controls). Only rats that would receive extinction underwent fear conditioning on day 0 . Tone controls remained in their home cages. CUS began the day after conditioning. Extinction was conducted on day 17, 3 days after the end of CUS. Tone controls were also exposed to context $\mathrm{B}$ and the presentation of tones, but as they had not been fear-conditioned, this produced no extinction learning. All rats were tested on the AST on day 18 (Figure 1e).

\section{Experiment 3: Effect of Extinction Training on the CUS-Induced Shift from Active to Passive Coping Behavior on the SPDB Test}

A total of 47 rats were used in 4 groups, defined by Stress (CUS or control) and Extinction (extinction or tone control). Only rats that would receive extinction underwent fear conditioning. CUS or nonstress control treatment began the day after fear conditioning. Extinction and tone control treatments were as above, 3 days after the end of CUS. All rats were tested on the SPDB test on day 18 (Figure 2a).

Shock-probe defensive burying test. The SPDB test was conducted as previously described (Roth et al, 2012). Behavior was recorded to video for 15 min following delivery of a single $2 \mathrm{~mA}$ shock upon first contact with the probe. Immobility and time spent actively burying the probe were scored from video by experimenters blind to treatment 

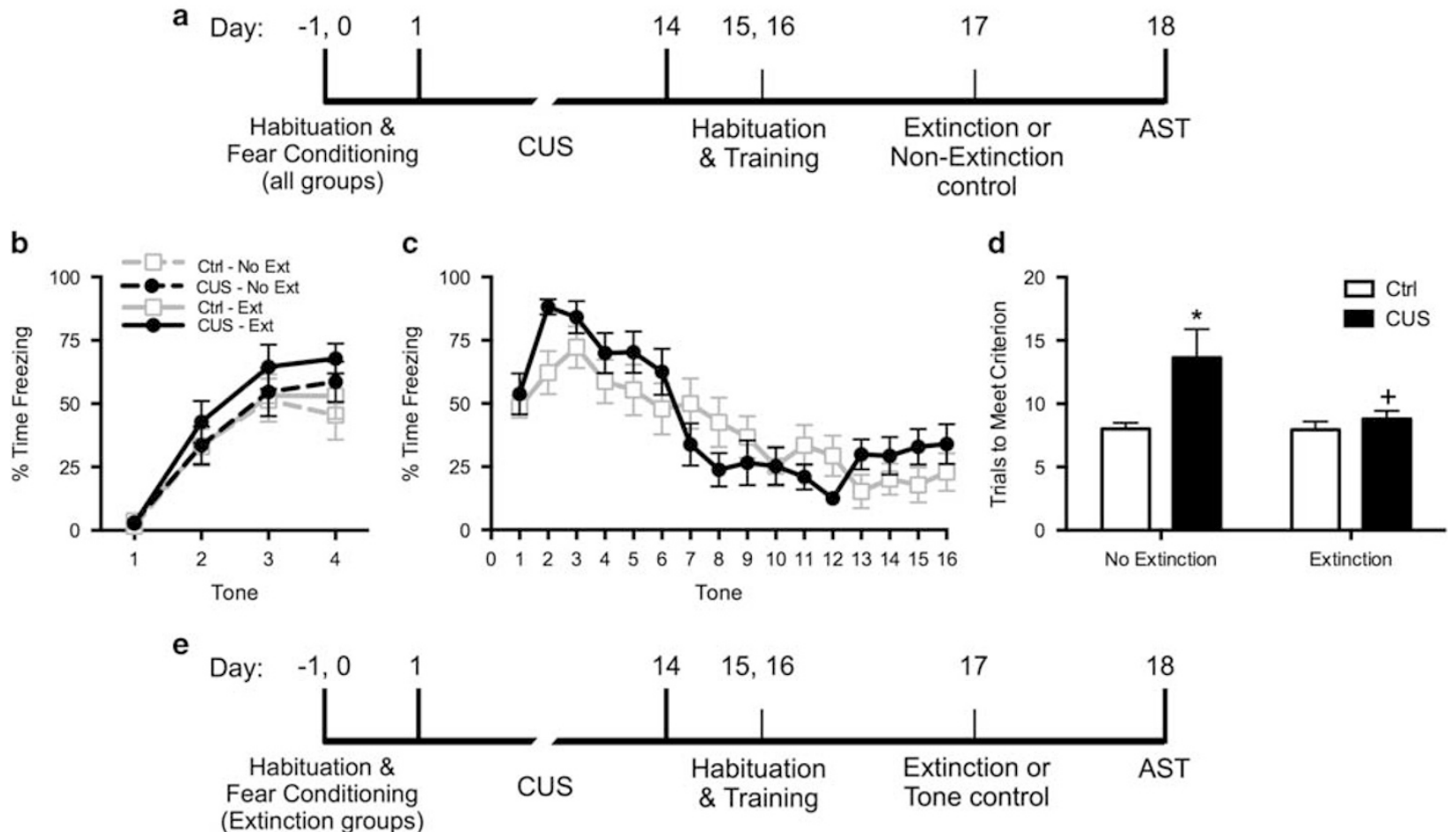

Cus
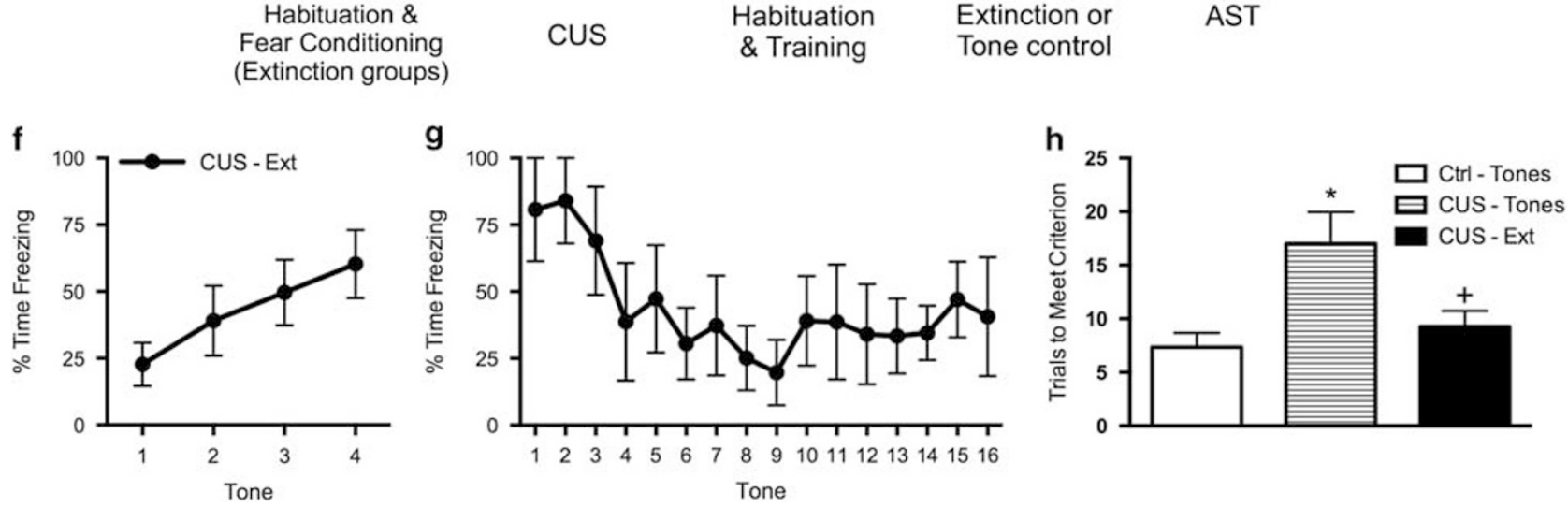

Figure I (a) Timeline for experiment I. (b) All rats were fear-conditioned and assigned to groups such that the groups had similar levels of freezing before beginning stress or control treatments. (c) The two extinction treatment groups (CUS and unstressed control) received a single extinction training session on day 17,24 h before testing on the attentional set-shifting test (AST). Extinction was comparable in the two groups (area under the curves, $p>0.65$ ); $n=14$ per group. (d) Extinction treatment reversed the chronic stress-induced deficit in cognitive flexibility on the extradimensional set-shifting task of the AST. CUS induced a significant increase in trials required to meet criterion (TTC) on the set-shifting task $(* p<0.05$, CUS compared with unstressed controls in the nonextinction groups). Extinction treatment reversed the effect of stress, restoring TTC to unstressed control levels $\left({ }^{+} p<0.05\right.$, extinction treatment compared with nonextinction in the CUS groups); $n=14-15$ per group. (e) Timeline for experiment 2, for which the CUS procedure included footshock. $(f)$ In experiment 2, the group undergoing fear conditioning displayed freezing behavior comparable to that seen in experiment I. Note that in experiment 2 , tone controls were exposed to the extinction procedure but were not fear-conditioned. (g) Extinction treatment was administered on day 17, $24 \mathrm{~h}$ before testing on the AST. Extinction was comparable to that seen in experiment I. The two tone control groups that were not fear-conditioned showed comparably low levels of freezing during tone presentation (not shown). (h) Again, extinction treatment reversed the chronic stress-induced deficit in cognitive flexibility on the extradimensional set-shifting task of the AST. CUS induced a significant increase in TTC on the set-shifting task $(* p<0.05$, CUS tone controls compared with unstressed tone controls). Extinction treatment reversed the effect of stress, restoring TTC to unstressed control levels ( ${ }^{+} p<0.05$, CUS-extinction compared with CUS-tone controls); $n=3-4$ per group. In all panels, data are expressed as mean \pm SEM.

groups. The measure of active relative to passive coping was the bury ratio, calculated as (bury time)/(bury time +immobility time).

\section{Experiment 4: Effect of Extinction Training on Phosphorylation of Ribosomal Protein S6 in the mPFC and LS of Chronically Stressed and Unstressed Rats}

A total of 38 rats were used in 6 groups, defined by Group (unstressed-extinction; CUS-extinction; or CUS-tone control) and Time (baseline or after extinction). Rats that were to undergo extinction were fear-conditioned. CUS or nonstress control treatment began the day after conditioning. Baseline rats were killed without exposure to the extinction procedure on day 17 . All others were killed immediately after completing the $32 \mathrm{~min}$ extinction procedure (Figure 3a). mPFC tissue was collected to measure changes in phosphorylated ribosomal protein S6 (pS6) that may be associated with activity known to be induced by extinction in this region. Tissue from LS, which mediates burying on 

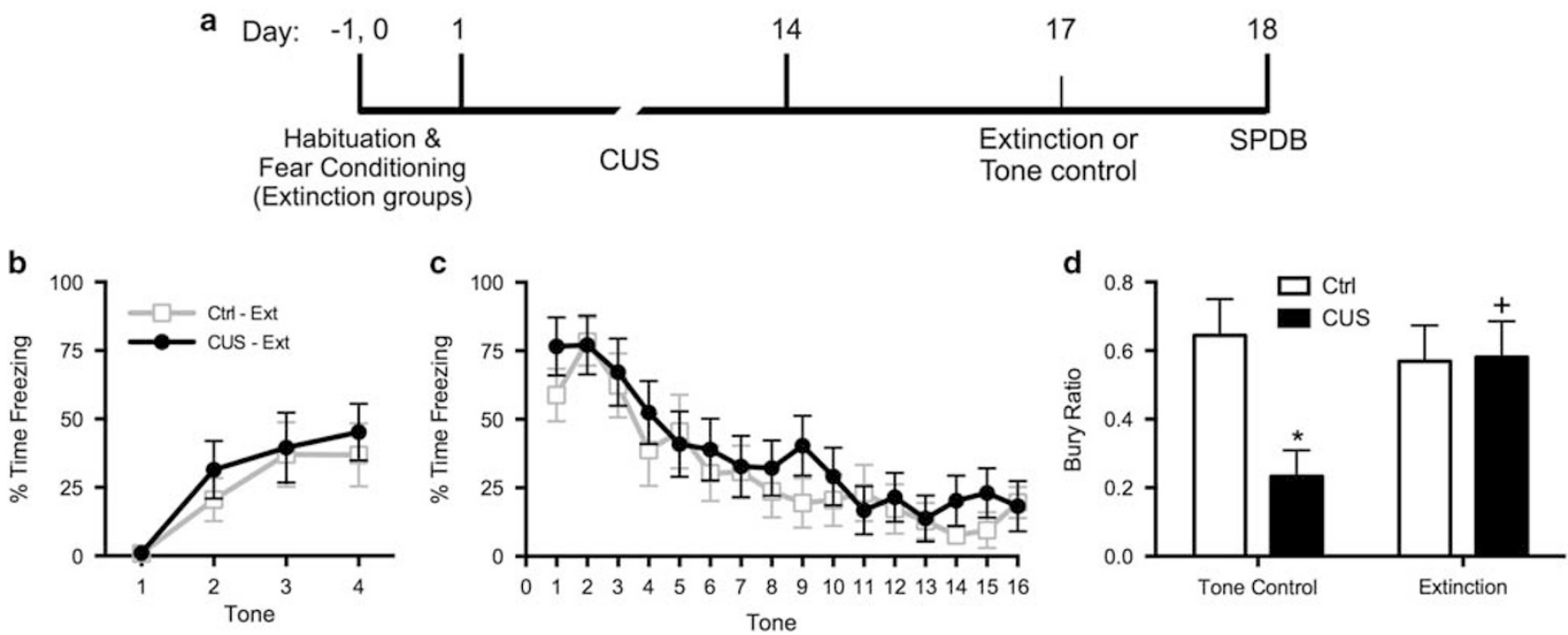

Figure 2 (a) Timeline for experiment 3. (b) Groups that underwent fear conditioning had similar levels of freezing before beginning stress or control treatments. (c) Extinction treatment was administered on day 17,24 h before testing on the shock probe test. Extinction was comparable in the two extinction treatment groups (CUS and unstressed control; area under the curves, $p>0.55$ ); $n=12$ per group. The two tone control groups that were not fearconditioned showed comparably low levels of freezing during tone presentation (not shown). (d) Extinction treatment reversed the chronic stress-induced shift from active to passive coping behavior on the shock probe defensive burying test. Chronic unpredictable stress (CUS) induced a significant decrease in the bury ratio (calculated as bury time/(bury time+immobility time); $* 0<0.05$, CUS tone controls compared with unstressed tone controls). Extinction treatment reversed the effect of stress, restoring the bury ratio to unstressed control levels $\left({ }^{+} p<0.05\right.$, CUS-extinction compared with CUS-tone controls); $n=11-12$ per group. In all panels, data are expressed as mean \pm SEM.

the SPDB test, was collected to compare mPFC to a brain region not known to be activated directly by extinction, but which is a downstream target of mPFC, the modulation of which may underlie shifts in coping behavior.

Western blots. Rats were killed by rapid decapitation. Brains were removed and $\mathrm{mPFC}$ and LS dissected on ice. For $\mathrm{mPFC}$, cortex adjacent and medial to the forceps minor was dissected from a $2 \mathrm{~mm}$ coronal slab cut $2-4 \mathrm{~mm}$ caudal to the frontal pole. For LS, septal tissue medial to the lateral ventricles was dissected from a $3 \mathrm{~mm}$ coronal slab cut 4-7 mm caudal to the frontal pole. Samples were stored at $-80^{\circ} \mathrm{C}$ until assay. Western blots were performed as previously described (Donegan et al, 2015). After transfer, membranes were incubated in polyclonal antibodies to pS6S240/244 $(1: 2000)$ and $\beta$-tubulin or $\beta$-actin $(1: 20000$, Cell Signaling, Beverly, MA) to normalize for loading, followed by anti-rabbit secondary antibody ( $1: 20000$, Cell Signaling), and detection with Prime ECL (GE Healthcare, Little Chalfont, UK). Blots were then stripped and reprobed with anti-S6 antibody ( $1: 1000$, Cell Signaling) followed by antimouse secondary antibody $(1: 10000)$. The ratio pS6/S6 was calculated for each sample, and relative expression of pS6 was computed for each subject as percent of mean baseline for that treatment group.

\section{Experiment 5: Effect of Blocking Protein Synthesis in the mPFC during Extinction Training on the Therapeutic Reversal of the CUS-Induced Deficit in Cognitive Flexibility on the AST}

A total of 44 rats were used in 6 groups (unstressedtone control-vehicle; CUS-tone control-vehicle; CUS-extinction-vehicle; CUS-extinction-anisomycin; unstressed-tone control-anisomycin; and CUS-extinction-anisomycin given in the PL). Rats were implanted stereotaxically with a guide cannula terminating $1 \mathrm{~mm}$ above the midline at the level of infralimbic (IL) cortex (from bregma: AP +2.9, ML +1.0, DV -4.1, angled $11^{\circ}$ laterally; Paxinos and Watson, 1986). This produces minimal damage to prelimbic cortex (PL) dorsal to IL, the region responsible for expression of conditioned fear. A site-specificity control group was implanted with a cannula targeting dorsal PL $(\mathrm{AP}+2.9$, ML +0.6, DV -2.1 , angled $11^{\circ}$ ). After 10 days of recovery, groups that were to undergo extinction were fear-conditioned. CUS or nonstress control treatment began the day after fear conditioning.

Microinjections. At 3 days after the end of CUS, on day 17, a microinjector extending $1 \mathrm{~mm}$ beyond the cannula tip was inserted, placing it at the midline between the two IL (Santini et al, 2004). Anisomycin $(50 \mu \mathrm{g} / 0.5 \mu \mathrm{l})$ or saline vehicle was injected at a rate of $0.1 \mu \mathrm{l} / \mathrm{min}$. This concentration of anisomycin blocked $>90 \%$ of protein synthesis for at least $2 \mathrm{~h}$ (Rosenblum et al, 1993), and impaired extinction retention without disrupting extinction behavior after injection into IL (Mueller et al, 2008; Santini et al, 2004). The injector remained in place $2 \mathrm{~min}$ before removing. At 20 min after injection, rats underwent extinction or tone control treatment. All rats were tested on the AST on day 18 (Figure 4a). After the experiment, cannula placement was confirmed histologically (Figure $4 \mathrm{~b}$ ).

Immunohistochemistry. To verify that anisomycin blocked protein synthesis, cFos expression was analyzed in a separate cohort of six rats in two groups (vehicle-extinction or anisomycin-extinction). Rats were injected with vehicle or anisomycin $20 \mathrm{~min}$ before extinction. At $1 \mathrm{~h}$ after completing extinction, rats were perfused and immunohistochemistry 

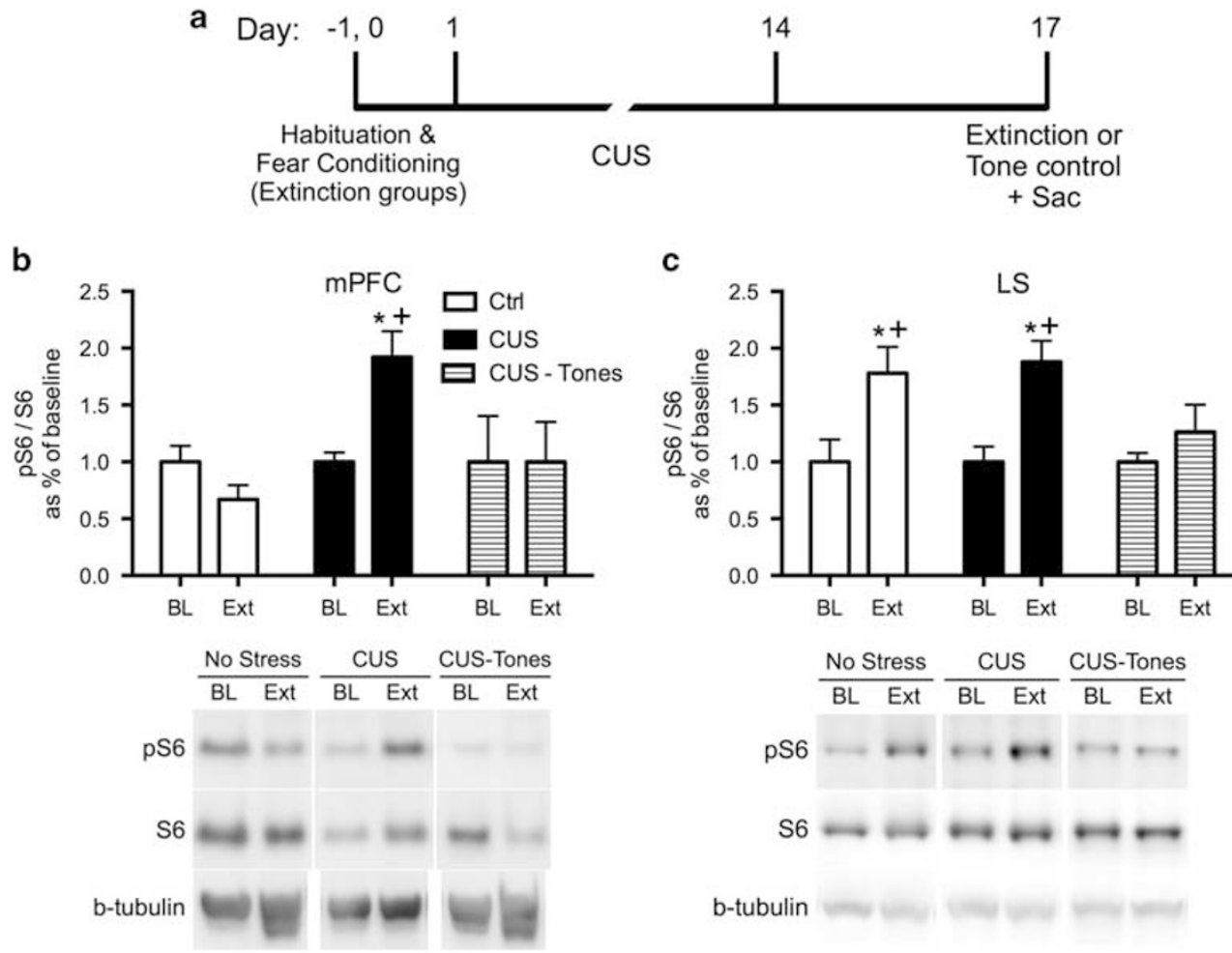

Figure 3 Extinction treatment increased phosphorylation of ribosomal protein S6 (pS6) in the medial prefrontal cortex (mPFC) and lateral septum (LS) of chronically stressed rats. (a) Timeline for experiment 4. All rats were killed on day 17 (Sac). Baseline groups were killed without extinction treatment, and the extinction groups were killed immediately after completing the 32 min extinction training session. (b) Extinction induced a significant increase in pS6 in the mPFC of CUS rats ( $* p<0.05$, Extinction compared with Baseline) but not in unstressed controls, nor in CUS-tone control rats exposed to tone presentations but without prior fear conditioning ( ${ }^{+} p<0.05$, CUS Extinction compared with unstressed Extinction and to CUS tone controls); $n=4-6$ per group. (c) Extinction also induced a significant increase in pS6 in the LS of unstressed and CUS rats (* $p<0.05$, Extinction compared with Baseline), that was not seen in CUS tone control rats ( $p=0.59$ compared with Baseline); $n=5-8$ per group. In all panels, data are expressed as mean \pm SEM.

performed on free-floating $40 \mu \mathrm{m}$ sections. After peroxidase inactivation, sections were incubated in primary cFos antibody (1:5000, Millipore, Temecula, CA) followed by HRP-conjugated anti-rabbit secondary antibody $(1: 2000$, Cell Signaling) and then fluorescein-tagged tyramide reagent (PerkinElmer, Waltham, MA). Fos-positive cells in IL were counted in a standard $200 \mu \mathrm{m}^{2}$ field in three sections per rat.

\section{Statistical Analyses}

Data were analyzed by one- or two-way ANOVA, with the exception of the cFos data in experiment 5 that were analyzed by $t$-test. Pairwise comparisons to detect specific group differences were performed using Newman-Keuls test. Significance was determined at $p<0.05$.

\section{RESULTS}

\section{Experiment 1: Effect of Extinction Training as a Therapeutic Intervention on the CUS-Induced Deficit in Cognitive Flexibility on the AST}

Fear conditioning was comparable in all groups before CUS or control treatment (Figure 1b). Similarly, the decrease in freezing was comparable in the two groups that underwent extinction (area under the curves, AUC: $t_{27}=0.5, p>0.65$; Figure 1c). Two-way ANOVA for effects on cognitive flexibility on the ED task revealed significant main effects of Stress $\left(\mathrm{F}_{1,53}=7.0, p<0.02\right)$ and Extinction $\left(\mathrm{F}_{1,53}=4.0\right.$, $p=0.05$ ). Newman-Keuls test revealed that CUS compromised cognitive flexibility, increasing TTC compared with unstressed controls ( $p<0.05$, Figure 1d), replicating previous findings (Bondi et al, 2008, 2010; Jett and Morilak, 2013). Extinction training $24 \mathrm{~h}$ before testing significantly reduced TTC in CUS rats compared with those that did not receive extinction $(p<0.05)$, restoring ED performance to control levels (Figure 1d).

Experiment 2: Replication of the Therapeutic Effect of Extinction Training on Cognitive Flexibility Using CUS with Footshock and Using Controls Exposed to the Extinction Chamber and Tones

The CUS-extinction group exhibited freezing behavior during conditioning and extinction comparable to experiment 1 (Figure if and g). Tone control rats that had not undergone fear conditioning on day 0 showed consistently low freezing during tone presentations on day 17 $(24.5 \pm 3.1 \%$ across all tones, mean \pm SEM, not shown).

One-way ANOVA revealed a significant effect on cognitive flexibility $\left(\mathrm{F}_{2,8}=5.4, p<0.05\right)$. Newman-Keuls test revealed that CUS again compromised cognitive flexibility, increasing TTC on the ED task $(p<0.05)$. Extinction training $24 \mathrm{~h}$ before testing again significantly reduced TTC in CUS rats 
a
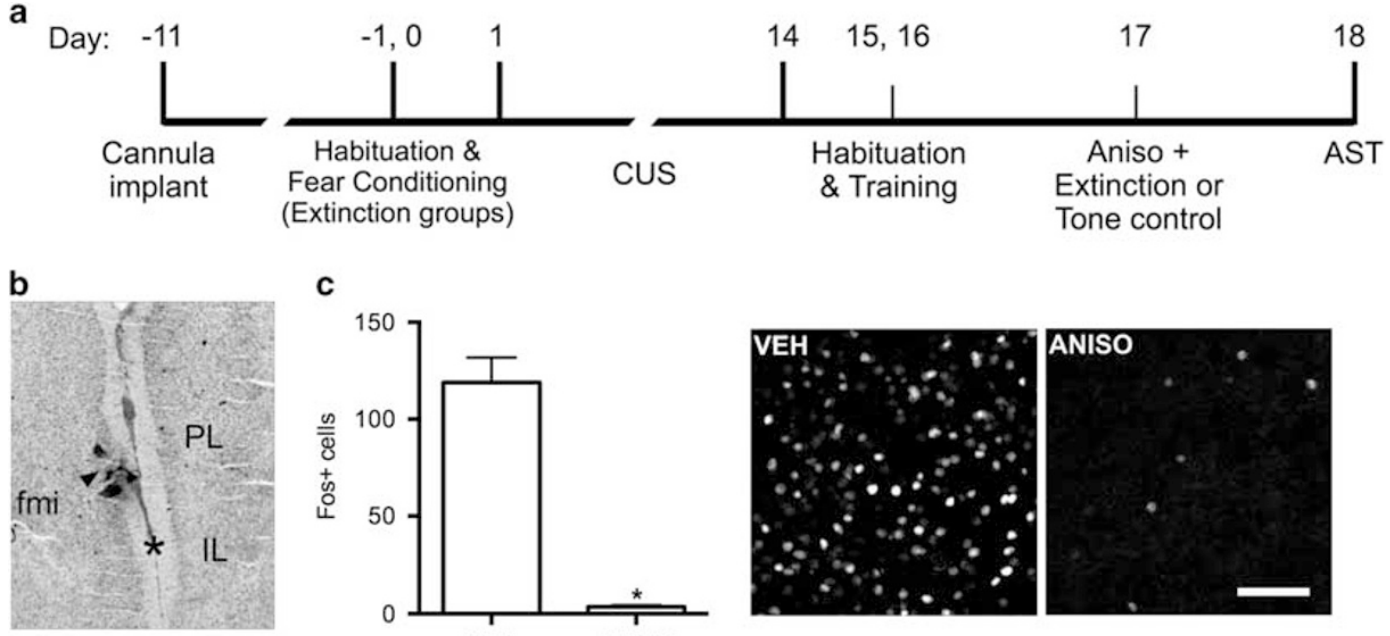

C
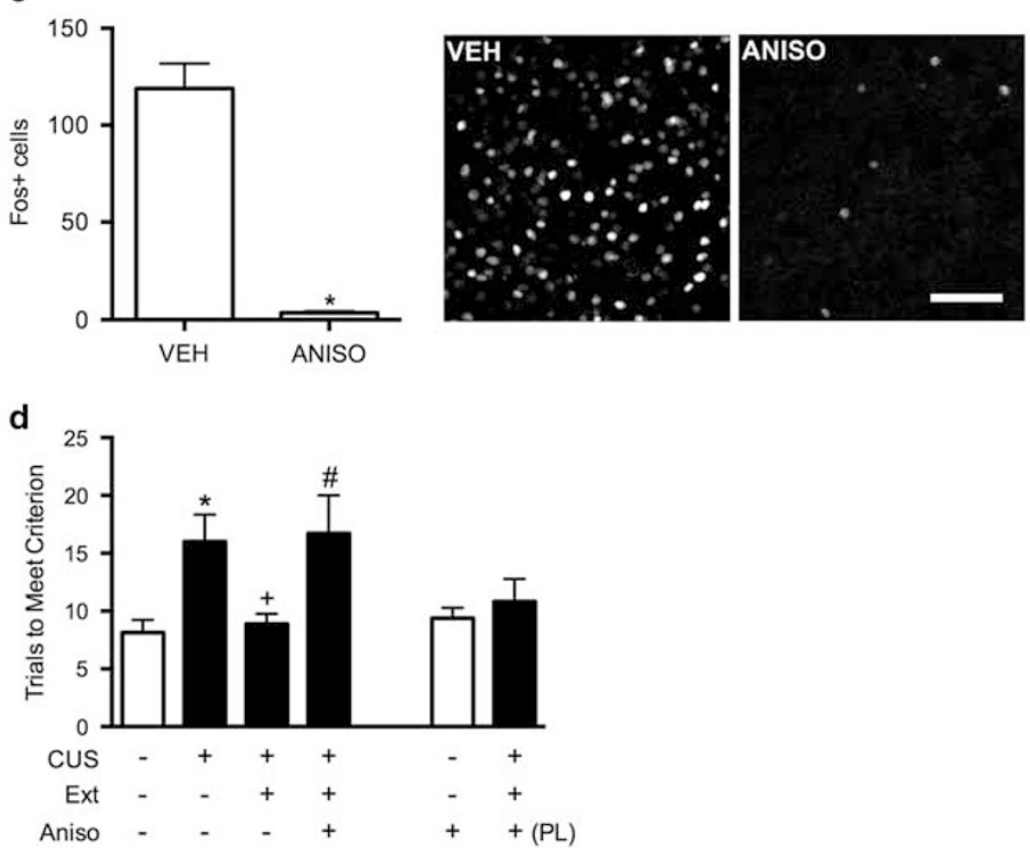

Figure 4 The beneficial effect of extinction after CUS requires protein synthesis in the ventral mPFC. (a) Timeline for experiment 5. (b) Interhemispheric injection site targeting infralimbic (IL) cortex. Arrow indicates cannula track at the point of penetration into the interhemispheric space at the level of IL (asterisk). Micrograph corresponds to plate 8 in Paxinos and Watson (I986). IL, infralimbic cortex; PL, prelimbic cortex; fmi, forceps minor. (c) In a separate cohort of rats, anisomycin microinjected into the IL cortex 20 min before extinction inhibited the induction of cFos protein expression in IL cortex I h after completion of extinction training ( $* 2<0.0002$ ); $n=3$ per group; scale bar $=50 \mu \mathrm{m}$. (d) Inhibition of protein synthesis in the IL cortex during extinction treatment prevented the rescue of cognitive set-shifting that had been compromised by CUS. CUS induced a significant increase in trials to criterion ( $T T C$ ) on the set-shifting task ( $p<0.05$, CUS-tone control-vehicle compared with unstressed-tone control-vehicle). Extinction treatment reversed the effect of stress, restoring TTC to unstressed control levels $\left({ }^{+} p<0.05\right.$, CUS-extinction-vehicle compared with CUS-tone control-vehicle). Microinjection of anisomycin into IL cortex before extinction treatment prevented the beneficial effect of extinction on set-shifting compromised by CUS, as TTCS were comparable to CUS tone controls ( ${ }^{*} p<0.05$, CUS-extinction-anisomycin compared with CUS-extinction-vehicle); $n=6-8$ per group. Administering anisomycin into the IL cortex of unstressed animals had no effect on set-shifting $24 \mathrm{~h}$ later. Similarly, as a site-specificity control, administering anisomycin into the prelimbic (PL) cortex of stressed animals before extinction did not prevent the therapeutic effect of extinction. In all panels, data are expressed as mean \pm SEM.

compared with CUS-tone controls $(p<0.05)$, restoring ED performance to unstressed control levels (Figure 1h), replicating the findings in experiment 1.

\section{Experiment 3: Effect of Extinction Training on the CUS- Induced Shift from Active to Passive Coping Behavior on the SPDB Test}

Fear conditioning was comparable before CUS or control treatment (Figure 2b), and extinction was comparable in groups that received fear conditioning (AUC: $t_{22}=0.6$, $p>0.7$; Figure 2c). Tone controls showed consistently low freezing during tone presentations on day $17(8.0 \pm 1.7 \%)$. Analysis of the bury ratio in the SPDB test revealed a significant Stress $\times$ Extinction interaction $\left(\mathrm{F}_{1,43}=4.5\right.$, $p<0.04)$, but no main effects. Newman-Keuls test revealed that CUS decreased the bury ratio of tone control rats $(p<0.05)$, reflecting a shift from active coping (burying) to passive coping (immobility). Extinction restored the bury ratio in CUS rats back to control levels (Figure 2d).

\section{Experiment 4: Effect of Extinction Training on Ribosomal Protein S6 Phosphorylation in the mPFC and LS of Chronically Stressed and Unstressed Rats}

Fear conditioning and extinction were comparable in the two groups receiving extinction treatment (AUC for extinction: $\left.t_{8}=0.4, p>0.1\right)$. Tone controls exhibited consistently low freezing during tone presentations on day $17(25.6 \pm 1.6 \%)$.

Figure $3 \mathrm{~b}$ shows phosphorylation of $\mathrm{pS} 6$ as a proportion of total S6 in the mPFC of rats killed immediately after fear extinction compared with baseline. Two-way ANOVA revealed a significant effect of Group $\left(\mathrm{F}_{2,22}=3.7, p<0.05\right)$ and a Group $\times$ Time interaction $\left(\mathrm{F}_{2,22}=3.7, p<0.05\right)$. Newman-Keuls test revealed that pS6 as a proportion of total S6 was significantly increased in the MPFC of CUS rats 
immediately after extinction compared with both the unstressed extinction group and the CUS tone control group, and with their baseline group $(p<0.05)$. There were no differences between groups for total S6 in the mPFC ( $p>0.1$, not shown).

Figure $3 \mathrm{c}$ shows pS6 in the lateral septum after extinction. Two-way ANOVA revealed a significant effect of extinction $\left(\mathrm{F}_{1,32}=17.3, p<0.001\right)$. Newman-Keuls test revealed that pS6 was significantly elevated in the LS of both unstressed and CUS rats after extinction compared with their respective baselines $(p<0.05)$. Tone controls showed no change in pS6 in the LS. There were no differences in total S6 in the LS $(p>0.1)$.

\section{Experiment 5: Effect of Blocking Protein Synthesis in the $\mathrm{mPFC}$ during Extinction Training on the Therapeutic Reversal of the CUS-Induced Deficit in Cognitive Flexibility on the AST}

cFos induction was inhibited in rats that received anisomycin in IL before extinction $\left(t_{4}=12.6, p=0.0002\right.$, Figure $\left.4 c\right)$, confirming the efficacy of anisomycin treatment. In the groups tested behaviorally, fear conditioning was comparable before CUS or control treatment, and extinction was comparable in the groups that were fear-conditioned (AUC: $F_{2,18}=0.6$, $p>0.5)$. Tone controls showed consistently low freezing during tone presentations on day $17(29.3 \pm 2.0 \%)$.

One-way ANOVA revealed a significant effect on cognitive flexibility $\left(\mathrm{F}_{5,38}=4.1, \quad p<0.01\right)$. Newman-Keuls test revealed that CUS again compromised cognitive flexibility, increasing TTC on the ED task $(p<0.05)$. Extinction training $24 \mathrm{~h}$ before testing again reduced TTC in CUS rats compared with CUS-tone controls $(p<0.05)$, restoring ED performance to unstressed control levels, replicating the findings in experiments 1 and 2. The reduction in TTC in CUS rats was prevented by anisomycin administration into IL before extinction training $(p<0.05)$, but not by anisomycin delivered into PL. Anisomycin given to unstressed rats on day 17 did not impair set-shifting performance on day 18 (Figure 4d).

\section{DISCUSSION}

This study demonstrated the utility of cue-conditioned fear extinction as a therapeutic intervention to model exposure therapy in rats. Extinction treatment ameliorated the CUSinduced deficit in MPFC-mediated cognitive flexibility on the AST, and the shift from active to passive coping behavior on the SPDB test. Moreover, the beneficial effect of extinction was evident $24 \mathrm{~h}$ after treatment, suggesting that plasticity in the circuitry underlying extinction accounted for these effects. Consistent with this, extinction induced phosphorylation of ribosomal protein S6 in the mPFC and LS of stressed animals, indicating activity-dependent changes in protein synthesis in these regions. The importance of this was further demonstrated by the finding that inhibiting protein synthesis in the IL during extinction blocked its therapeutic effect on cognitive flexibility in stressed animals.

Validity of extinction as a model of exposure therapy. The basis for considering exposure therapy as an extinction process has been noted previously (Hoffmann, 2008; McNally, 2007), and this model has face validity. Before extinction, through repeated association of an innocuous tone with an aversive footshock, rats learn that the cue predicts 'danger' and it becomes aversive itself, eliciting a stress response and fear behavior. For extinction then, by exposing the animals in a different context to repeated presentations of the tone alone without shock, they learn that in that context the cue no longer predicts danger, and the fear response is suppressed. Similarly, in PTSD, cues become conditioned to elicit an intense stress response by association with fearful stimuli. This is adaptive for survival in a lifethreatening environment, but upon return to a safe environment, those same responses to cues that no longer signal danger are maladaptive. In exposure therapy, as in extinction, patients are repeatedly exposed, in a safe environment, to cues that have been previously associated with fearful stimuli. They gradually learn that in this context, those cues are not associated with anticipated danger, and fearful responses elicited by those stimuli are reduced (McLean and Foa, 2013). Thus, extinction and exposure therapy are both forms of cognitive flexibility in which expectations of negative outcome predicted by cues previously associated with an adverse event are modified by changing environmental feedback (Hoffmann, 2008). Importantly, this modification of negative expectations is central to cognitive behavioral therapies used to treat a range of stress-related psychiatric illnesses, not just exposure therapy for PTSD. Our results demonstrate that extinction improves stress-compromised cognitive flexibility, targeting a common cognitive dimension underlying these illnesses. Exposure therapy can also be used to treat disorders like depression and prolonged grief that are not fear related (Bryant et al, 2014; Hayes et al, 2005), implicating extinction as a useful model of effective cognitive behavioral psychotherapy across illnesses that share a similar cognitive component.

Extinction as a model of exposure therapy also has construct validity. Fear conditioning occurs in the basolateral amygdala. Extinction learning requires the ventral mPFC, specifically IL cortex in rats, that suppresses the activity and output of the amygdala, whereas expression of conditioned fear requires the more dorsal PL subregion of $\mathrm{mPFC}$ (SotresBayon et al, 2004). In stress-related psychiatric illnesses like depression and PTSD, the amygdala is hyperexcitable and the ventral $\mathrm{mPFC}$ is hypoactive (Drevets, 2000; Elzinga and Bremner, 2002), whereas patients undergoing effective psychotherapy show elevated activity in $\mathrm{mPFC}$ and reduced reactivity in the amygdala (Ritchey et al, 2011).

Potential circuitry underlying the therapeutic effects of extinction learning. The role of $\mathrm{mPFC}$ in extinction is well established (Quirk et al, 2006). Thus, extinction training may enhance mPFC function, exerting a direct beneficial effect on other measures of cognitive flexibility mediated in the mPFC. Furthermore, in addition to improving cognitive flexibility, another goal of cognitive behavioral therapy is to improve adaptive coping, and extinction also restored active coping behavior on the SPDB after CUS. It is possible that enhancing $\mathrm{mPFC}$ function could secondarily modulate the activity of subcortical limbic regions such as the LS via a top- 
down mechanism. However, extinction may also influence the function of the LS directly. The LS has a complex role in fear conditioning and plasticity related to both the expression and suppression of fear-related behaviors (Sheehan et al, 2004). Thus, the observation that extinction increased pS6 in the LS, particularly in unstressed rats, which was not seen in $\mathrm{mPFC}$, may suggest that extinction directly enhances the function of this brain region, in addition to secondary modulation by the mPFC. Whether this implies that LS is involved in mediating any aspect of extinction learning itself requires further investigation.

The role of prefrontal protein synthesis in the therapeutic effects of extinction. The hypothesis that protein synthesis is required for the long-term therapeutic effects of extinction in stress-compromised animals was directly supported by the observation that administering anisomycin into the mPFC at the time of extinction blocked its therapeutic effect on cognitive flexibility the next day. Increased phosphorylation of ribosomal protein S6 was not seen in the mPFC of unstressed rats following extinction, consistent with a previous report (Tedesco et al, 2014). However, pS6 was increased in the mPFC of stressed rats after extinction, suggesting that different cellular processes are engaged by extinction in a stress-compromised $\mathrm{mPFC}$ that are not engaged in control mPFC. The significance of S6 phosphorylation for protein translation is unclear (Biever et al, 2015). It is not required for protein synthesis per se, but changes in pS6 have been associated with changes in the overall level of protein synthesis, and also with increased neural activity, prompting the use of pS6 as a marker of activity-dependent changes in protein synthesis (Knight et al, 2012; see Biever et al, 2015). Lack of pS6 induction in unstressed animals does not imply a lack of protein synthesis that is known to occur after extinction. Rather, induction of pS6 may indicate that specific proteins are translated in the mPFC of stressed animals that are not translated in control animals, and these may contribute to the beneficial effect of extinction on cognitive flexibility that was only seen in stressed rats. The specific factors translated after S6 phosphorylation that might influence cognitive flexibility are not known. Nonetheless, the importance of these processes to therapeutic efficacy is supported by reports that signaling pathways leading to S6 phosphorylation and initiation of protein synthesis (eg, the mTOR-p70S6K pathway responsible for phosphorylation of S6 at S240/244) are also induced by acute administration of a low dose of ketamine, another novel therapeutic intervention that produces rapid, lasting effects after single treatment (Duman et al, 2012).

Summary. These results establish the utility of fear extinction as a therapeutic behavioral intervention that models exposure therapy, a form of cognitive behavioral psychotherapy. They reveal molecular changes in brain regions relevant to the behavioral dimensions of stressrelated psychiatric illness that may underlie the beneficial effects of extinction on cognition and coping behavior compromised by chronic stress. Increased phosphorylation of ribosomal protein $\mathrm{S} 6$ in the $\mathrm{mPFC}$ of stressed rats and blockade of the therapeutic effect of extinction by anisomycin administration into the mPFC indicate that extinction- induced protein translation underlies the beneficial effects of extinction, and perhaps similarly of exposure therapy. More generally, this model provides a preclinical platform for further exploration of neurobiological mechanisms underlying the efficacy of psychotherapy that may suggest new targets for pharmacological intervention.

\section{FUNDING AND DISCLOSURE}

Dr DA Morilak serves on the Psychopharmacology advisory board for $\mathrm{H}$. Lundbeck A/S and receives research funding from Lundbeck Research USA. These activities have no relation to any of the work presented in this paper. The other authors declare no conflict of interest.

\section{ACKNOWLEDGMENTS}

We thank Lauren Hatherall, Jeri Silva, Suphada Lertphinyowong, and Kathleen Winters for technical assistance. This work was supported by Research Grant MH072672 from the National Institute of Mental Health, T32 Training Grant NS082145 from the National Institutes of Health, a fellowship awarded through Grant TL1 TR001119 from the National Center for Advancing Translational Sciences, National Institutes of Health, and a grant from the William and Ella Owens Medical Research Foundation, none of which had any role in study design, data collection, analysis or interpretation, or in the preparation or decision to submit this paper for publication.

\section{AUTHOR CONTRIBUTIONS}

EA Fucich participated in experimental design and planning, conduct of the experiments, data analysis and interpretation, and writing and editing the manuscript. D Paredes participated in conduct of the experiments, data analysis, and editing of the manuscript. DA Morilak participated in experimental design and planning, data analysis and interpretation, writing and editing the manuscript, and provided laboratory resources and oversight for the experiments. All authors have read and approved the final manuscript.

\section{REFERENCES}

Beck AT (1976). Cognitive Therapy and the Emotional Disorders. Int. Univ. Press: New York.

Beck AT (2005). The current state of cognitive therapy: a 40-year retrospective. Arch Gen Psychiatry 62: 953-959.

Biehn TL, Contractor A, Elhai JD, Tamburrino M, Fine TH, Prescott MR et al (2013). Relations between the underlying dimensions of PTSD and major depression using an epidemiological survey of deployed Ohio National Guard soldiers. J Affective Disord 144: 106-111.

Biever A, Valjent E, Puighermanal E (2015). Ribosomal protein S6 phosphorylation in the nervous system: From regulation to function. Front Mol Neurosci 8: 75.

Birrell JM, Brown VJ (2000). Medial frontal cortex mediates perceptual attentional set shifting in the rat. J Neurosci 20: 4320-4324.

Bondi CO, Barrera G, Lapiz MDS, Bédard T, Mahan A, Morilak DA. (2007). Noradrenergic facilitation of shock-probe 
defensive burying in lateral septum of rats, and modulation by chronic treatment with desipramine. Prog Neuropsychopharmacol Biol Psychiatry 31: 482-495.

Bondi CO, Jett JD, Morilak DA (2010). Beneficial effects of desipramine on cognitive function of chronically stressed rats are mediated by alpha1-adrenergic receptors in medial prefrontal cortex. Prog Neuropsychopharmacol Biol Psychiatry 34: 913-923.

Bondi CO, Rodriguez G, Gould GG, Frazer A, Morilak DA (2008). Chronic unpredictable stress induces a cognitive deficit and anxietylike behavior in rats that is prevented by chronic antidepressant drug treatment. Neuropsychopharmacology 33: 320-331.

Brewin CR (1996). Theoretical foundations of cognitivebehavior therapy for anxiety and depression. Annu Rev Psychol 47: 33-57.

Bryant RA, Kenny L, Joscelyne A, Rawson N, Maccallum F, Cahill C et al (2014). Treating prolonged grief disorder: a randomized clinical trial. JAMA Psychiatry 71: 1332-1339.

Buffington SA, Huang W, Costa-Mattioli M (2014). Translational control in synaptic plasticity and cognitive dysfunction. Annu Rev Neurosci 37: 17-38.

Coles ME, Heimberg RG (2002). Memory biases in the anxiety disorders: current status. Clin Psychol Rev 22: 587-627.

de Kleine RA, Rothbaum BO, van Minnen A (2013). Pharmacological enhancement of exposure-based treatment in PTSD: a qualitative review. Eur J Psychotraumatol 4: 21626.

Donegan JJ, Patton MS, Chavera TA, Berg KA, Morilak DA, Girotti $\mathrm{M}$ et al (2015). Interleukin-6 attenuates serotonin2A receptor signaling by activating the JAK-STAT pathway. Mol Pharmacol 87: 492-500.

Drevets WC (2000). Functional anatomical abnormalities in limbic and prefrontal cortical structures in major depression. Prog Brain Res 126: 413-431.

Duman RS, Li N, Liu R-J, Duric V, Aghajanian G (2012). Signaling pathways underlying the rapid antidepressant actions of ketamine. Neuropharmacology 62: 35-41.

Elhai JD, Carvalho LF, Miguel FK, Palmieri PA, Primi R, Christopher Frueh B (2011). Testing whether posttraumatic stress disorder and major depressive disorder are similar or unique constructs. J Anxiety Disord 25: 404-410.

Elzinga BM, Bremner JD (2002). Are the neural substrates of memory the final common pathway in posttraumatic stress disorder (PTSD)? J Affect Disord 70: 1-17.

Foa EB, Meadows EA (1997). Psychosocial treatments for posttraumatic stress disorder: a critical review. Ann Rev Psychol 48: 449-480.

Green MK, Rani CSS, Joshi A, Soto-Piña AE, Martinez PA, Frazer A et al (2011). Prenatal stress induces long term stress vulnerability, compromising stress response systems in the brain and impairing extinction of conditioned fear after adult stress. Neuroscience 192: 438-451.

Hayes AM, Beevers CG, Feldman GC, Laurenceau JP, Perlman C (2005). Avoidance and processing as predictors of symptom change and positive growth in an integrative therapy for depression. Int J Behav Med 12: 111-122.

Hoffmann SG (2008). Cognitive processes during fear acquisition and extinction in animals and humans: implications for exposure therapy of anxiety disorders. Clin Psychol Rev 28: $199-210$

Hollon SD, Thase ME, Markowitz JC (2002). Treatment and prevention of depression. Psychol Sci Public Interest 3: 39-77.

Janicak PG, Davis JM, Preskorn SH, Ayd FJ. Treatment with antidepressants. In: Janicak PG, Davis JM, Preskorn SH, Ayd FJ (eds). Principles and Practice of Psychopharmacotherapy, 2nd edn. Williams \& Wilkins: Baltimore, 1997, pp 243-356.

Jett JD, Boley AM, Girotti M, Shah A, Lodge DJ, Morilak DA (2015). Antidepressant-like cognitive and behavioral effects of acute ketamine administration associated with plasticity in the ventral hippocampus to medial prefrontal cortex pathway. Psychopharmacology 232: 3123-3133.

Jett JD, Morilak DA (2013). Too much of a good thing: blocking noradrenergic facilitation in medial prefrontal cortex prevents the detrimental effects of chronic stress on cognition. Neuropsychopharmacology 38: 585-595.

Knight ZA, Tan K, Birsoy K, Schmidt S, Garrison JL, Wysocki RW et al (2012). Molecular profiling of activated neurons by phosphorylated ribosome capture. Cell 151: 1126-1137.

Koolhaas JM, Korte SM, De Boer SF, Van der Vegt BJ, Van Reenen CG, Hopster H et al (1999). Coping styles in animals: current status in behavior and stress-physiology. Neurosci Biobehav Rev 23: 925-935.

Lapiz MDS, Morilak DA (2006). Noradrenergic modulation of cognitive function in rat medial prefrontal cortex as measured by attentional set shifting capability. Neuroscience 137: 1039-1049.

Mathews A, Mackintosh B (1998). A cognitive model of selective processing in anxiety. Cognit Ther Res 22: 539-560.

McLean CP, Foa EB (2013). Dissemination and implementation of prolonged exposure therapy for posttraumatic stress disorder. J Anxiety Disord 27: 788-792.

McNally RJ (2007). Mechanisms of exposure therapy: how neuroscience can improve psychological treatments for anxiety disorders. Clin Psychol Rev 27: 750-759.

Mueller D, Porter JT, Quirk GJ (2008). Noradrenergic signaling in infralimbic cortex increases cell excitability and strengthens memory for fear extinction. J Neurosci 28: 369-375.

Nemeroff CB, Helm CM, Thase ME, Klein DN, Rush AJ, Schatzberg AF et al (2003). Differential responses to psychotherapy versus pharmacotherapy in patients with chronic forms of major depression and childhood trauma. Proc Natl Acad Sci USA 100: 14293-14296.

Nutt DJ (2010). Rationale for, barriers to, and appropriate medication for the long-term treatment of depression. J Clin Psychiatry 71: e02.

Paxinos G, Watson C. The Rat Brain in Stereotaxic Coordinates, 2nd edn. Academic Press: San Diego, 1986.

Quirk GJ, Garcia R, González-Lima F (2006). Prefrontal mechanisms in extinction of conditioned fear. Biol Psychiatry 60: 337-343.

Richter JD, Klann E (2009). Making synaptic plasticity and memory last: mechanisms of translational regulation. Genes Dev 23: $1-11$.

Ritchey M, Dolcos F, Eddington KM, Strauman TJ, Cabeza R (2011). Neural correlates of emotional processing in depression: changes with cognitive behavioral therapy and predictors of treatment response. J Psychiatr Res 45: 577-587.

Rogers MA, Kasai K, Koji M, Fukuda R, Iwanami A, Nakagome K et al (2004). Executive and prefrontal dysfunction in unipolar depression: a review of neuropsychological and imaging evidence. Neurosci Res 50: 1-11.

Rosenblum K, Meiri N, Dudai Y (1993). Taste memory: the role of protein synthesis in gustatory cortex. Behav Neural Biol 59: 49-56.

Roth MK, Bingham B, Shah A, Joshi A, Frazer A, Strong R et al (2012). Effects of chronic plus acute prolonged stress on measures of coping style, anxiety, and evoked HPA-axis reactivity. Neuropharmacology 63: 1118-1126.

Santini E, Ge H, Ren K, Peña de Ortiz S, Quirk GJ (2004). Consolidation of fear extinction requires protein synthesis in the medial prefrontal cortex. J Neurosci 24: 5704-5710.

Shah AA, Sjuvold T, Treit D (2004). Inactivation of the medial prefrontal cortex with the $\mathrm{GABA}_{\mathrm{A}}$ receptor agonist muscimol increases open-arm activity in the elevated plus-maze and attenuates shock-probe burying in rats. Brain Res 1028: $112-115$.

Sheehan TP, Chambers RA, Russel DS (2004). Regulation of affect by the lateral septum: implications for neuropsychiatry. Brain Res Rev 46: 71-117. 
Sheline YI (2003). Neuroimaging studies of mood disorder effects on the brain. Biol Psychiatry 54: 338-352.

Sotres-Bayon F, Bush DE, LeDoux JE (2004). Emotional perseveration: an update on prefrontal-amygdala interactions in fear extinction. Learn Mem 11: 525-535.

Straub J, Plener PL, Sproeber N, Sprenger L, Koelch MG, Groen G et al (2015). Neural correlates of successful psychotherapy of depression in adolescents. J Affect Disord 183: 239-246.

Tedesco V, Roquet RF, DeMis J, Chiamulera C, Monfils MH (2014). Extinction, applied after retrieval of auditory fear memory, selectively increases zinc-finger protein 268 and phosphorylated ribosomal protein S6 expression in prefrontal cortex and lateral amygdala. Neurobiol Learn Mem 115: 78-85.
Treit D, Pesold C, Rotzinger S (1993). Dissociating the anti-fear effects of septal and amygdaloid lesions using two pharmacologically validated models of rat anxiety. Behav Neurosci 107: 770-785.

Wiles N, Thomas L, Abel A, Ridgway N, Turner N, Campbell J et al (2013). Cognitive behavioural therapy as an adjunct to pharmacotherapy for primary care based patients with treatment resistant depression: results of the CoBalT randomised controlled trial. Lancet 381: 375-384.

Yoshimura S, Okamoto Y, Onoda K, Matsunaga M, Okada G, Kunisato $\mathrm{Y}$ et al (2014). Cognitive behavioral therapy for depression changes medial prefrontal and ventral anterior cingulate cortex activity associated with self-referential processing. Soc Cogn Affect Neurosci 9: 487-493. 